\title{
Domino effects of COVID-19 in pregnancy:it's time for action
}

\author{
Rui-Hong Xue ${ }^{1}$ and Hefeng Huang ${ }^{2}$ \\ ${ }^{1}$ International Peace Maternity and Child Health Hospital, School of Medicine, Shanghai \\ Jiao Tong University, Shanghai, China \\ ${ }^{2}$ International Peace Maternity and Child Health Hospital, School of Medicine,
}

October 21, 2020

\section{Domino effects of COVID-19 in pregnancy:it's time for action}

Rui-Hong Xue ${ }^{1}$, He-Feng Huang ${ }^{2,3,4,5}$

${ }^{1}$ Department of Obstetrics and Gynecology, International Peace Maternity and Child

Health Hospital, School of Medicine, Shanghai Jiao Tong University, Shanghai, China

${ }^{2}$ Center of Reproductive Medicine, International Peace Maternity and Child Health Hospital, School of Medicine, Shanghai Jiao Tong University, Shanghai, China

${ }^{3}$ Institute of Embryo-Fetal Original Adult Disease Affiliated to Shanghai Jiao Tong University School of Medicine, Shanghai Jiao Tong University, Shanghai, China

${ }^{4}$ Shanghai Key Laboratory of Embryo Original Diseases, Shanghai, China

${ }^{5}$ Shanghai Municipal Key Clinical Specialty, Shanghai, China

Correspondence: He-feng Huang, E-mail:huanghefg@hotmail.com;Postal address: No.910 Hengshan Road, Shanghai, China. Postal code: 200030. Work phone: +86-021-64070434

The author report no conflict of interest.

Word Count: 674

Funding: None

COVID-19 is still spreading rapidly around the world, with millions of people having lost lives. Pregnant women represent a uniquely vulnerable group in any infectious disease outbreak because of their altered physiology, susceptibility to infections, and compromised mechanical and immunological functions. Though the management guidelines during pregnancy are evolving continuously, pregnant women suffered from COVID19 mean worse pregnancy outcomes, both physically and mentally. As is reported more distress and psychiatric symptoms the infected pregnant women would have than those who were assessed before the pandemic, mainly in the form of depression and anxiety symptoms. What was worse, COVID-19 may increase fetal complications including miscarriage (2\%), intrauterine growth restriction (IUGR; 10\%), and preterm birth (39\%). Pregnancy is the window for the future, the COVID-19 could result in short and long-term potential harmful to the neonate, including malformation, reproductive or sexual functions, or even intelligence. Early failures in prevention of the virus have resulted in Domino effect, and "it's very tough for countries right now in Europe and North America going back to another phase of potential lockdowns", as a WHO officer said. People are exhausted and very tired. However, there are still many people who refuse to have a mask at public around the world during the global pandemic. 
In pregnancy, the evaluations of the safety the mode of delivery and breast feeding mainly come from some case series. The limited sample size limited statistical comparison between groups. In a case series of 13 pregnant women with COVID-19, negative viral test results were observed in vaginal secretion specimens, suggesting that a vaginal delivery may be a safe delivery option. However, this case series did not consider the risks of respiratory transmission of the virus from the mother to the neonate during delivery or labor. Considering the above, an analyses of neonatal outcome in 29 pregnant women with COVID-19 were conducted, among which 12 of 13 hospitalized neonates presented with radiological features for pneumonia through X-ray or CT screening, and suggested that intrauterine or intrapartum transmission is possible. Although a systematic review concluded that the rate of infection is no greater when the baby is born vaginally, breastfed or remains with the mother, the safety of mode of delivery among COVID-19 infected pregnant women remains controversial and calls for prospective study.

Multiple COVID-19 vaccines are currently in phase 3 trials with efficacy assessed as prevention of virologically confirmed disease. Vaccines have been considered the promising way to effectively control the virus, however, the effectiveness and safety of vaccines or drugs to be used in pregnant women have been reported very few and need further investigations. As reported in an example that respiratory syncytial virus vaccination in pregnant women did not meet the prespecified success criterion for efficacy against RSV-associated, medically significant lower respiratory tract infection in infants up to 90 days of life. On the basis of available data, and in line with the precautionary principle, the risk of COVID-19 in pregnancy should not be downplayed to avoid falsely reassuring healthcare professionals and the public and women should be advised to take necessary precautions to avoid infection during pregnancy. However, it has been confirmed that public health interventions could improve the control of the COVID-19 outbreak. The risks of respiratory transmission of COVID-19 from the other to the neonate remain unclear and need further investigations. Before that, the best suggestion in a non-effected population is still physical distancing, face masks, and eye protection.

COVID-19 characters as strong infectivity, rapid and wide spread, and have caused significant morbidity and mortality. In the 1902 paper, Ballantyne said, "as with the premature, the ideal plan of procedure is prevention", the same principle with COVID-19. Before the assurance of the effectiveness and safety of COVID-19 vaccines to be used in pregnant women, strong actions of protection and prevention to be taken in the non-infected pregnant population is still the best suggestion. Only by this, can we see the turning point in the rising curve. The prevention of COVID-19 in pregnancy calls for action, better late than never.

\section{Contributions}

HF-H was responsible for the design and supervision of the work and have made critical revision. RH-X wrote the manuscript.

\section{Sources of funding}

None

\section{Disclosure of interests}

The authors of this work declare no potential conflicts of interest relevant to this article.

\section{Ethics approval}

The manuscript was approved by the ethics committee on human research. Review Board: (GKLW) 2020-05 On March 15, 2020. 